\title{
Factores de riesgo modificables de enfermedad cerebrovascular en pacientes que han sufrido un ictus
}

\author{
Modifiable risk factors of cerebrovascular disease in stroke patients \\ Fatores de risco modificáveis da doença cerebrovascular em pacientes \\ que tem sofrido um ictus
}

\author{
Eliana R. Meza-Miranda ${ }^{*}$, Nélida R. Romero-Espínola², Emma A. Báez Ortíz². \\ Recibido: 21 de junio de 2021. Aceptado para publicación: 28 de septiembre de 2021 . \\ Primero en línea: 29 de septiembre de 2021. \\ https://doi.org/10.35454/rncm.v4n4.317
}

\section{Resumen}

Introducción: el accidente cerebrovascular (ACV) es una enfermedad neurológica cuyo impacto a nivel de salud pública es de relevancia debido a su alta incidencia, mortalidad y grado de invalidez. El presente estudio tuvo como objetivo determinar la frecuencia de los factores de riesgo modificables en pacientes con ACV del Hospital Central de la Policía "Rigoberto Caballero".

Metodología: estudio observacional descriptivo de corte transversal en pacientes adultos con antecedente de ACV hospitalizados. Se evaluaron las variables de estado nutricional y factores de riesgo modificables (hipertensión arterial [HTA], dislipidemia, diabetes mellitus tipo 2 [DM2], tabaquismo y cardiopatías). Se muestran el promedio y las desviaciones estándar, según corresponda. Las variables cualitativas fueron expresadas en frecuencia $(n)$ y porcentaje (\%).

Resultados: se evaluó un total de 70 pacientes al ingreso, el $54 \%$ fue de sexo femenino y el promedio de edad fue de 69,1 $\pm 12,24$ años. El tipo de ACV predominante fue el isquémico $(84,3 \%)$ y la mayoría tenía un peso adecuado (34,3\%), seguido de sobrepeso $(31,4 \%)$. La mayoría presentó niveles deseables de colesterol total $(64,3$ $\%$ ), niveles bajos de colesterol HDL (lipoproteína de alta densidad; 91,4 \%), límite elevado de colesterol LDL (lipoproteína de baja densidad; $47,1 \%$ ) y niveles normales

\section{Summary}

Introduction: A cerebrovascular accident (CVA) is a neurological condition with significant impact on public health due to its high incidence, mortality and degree of disability. The aim of this study was to determine the frequency of modifiable risk factors in stroke patients at the Rigoberto Caballero Police Central Hospital.

Methodology: Cross-sectional descriptive observational study of hospitalized adult patients with a history of stroke. The variables assessed were nutritional status and modifiable risk factors (hypertension dyslipidemia, DM II, smoking and heart disease). Means and standard deviations are shown as appropriate. The qualitative variables were expressed in frequency $(n)$ and percentage (\%).

Results: A total of 70 patients were assessed on admission; 54\% were female, and mean age was $69.1 \pm 12.24$ years. The predominant type of stroke was ischemic (84.3\%). The majority of patients were of adequate weight (34.3\%), and $31.4 \%$ were overweight $(31.4 \%)$. The majority had desirable levels of total cholesterol (64.3\%), low levels of HDL cholesterol (91.4\%), LDL cholesterol in the upper limit (47.1\%) and normal triglyceride levels $(<150 \mathrm{mg} / \mathrm{dL}$, $38.5 \%)$. Regarding other risk factors, the majority had arterial hypertension (81.4\%).

Conclusion: In patients with a history of stroke, the predominant modifiable risk factor was elevated blood pressure,

\section{Resumo}

Introdução: O acidente vascular cerebral (AVC) é uma doença neurológica cujo impacto a nível de saúde pública é relevante devido a sua elevada incidência, mortalidade e grau de incapacidade. O objetivo deste estudo foi determinar a frequência dos fatores de risco modificáveis em pacientes com AVC do Hospital Central da Polícia Rigoberto Caballero.

Metodologia: Estudo transversal observacional descritivo em pacientes adultos hospitalizados com história de acidentes vasculares cerebrais. Foram avaliadas as variáveis: estado nutricional e fatores de risco modificáveis (hipertensão, dislipidemia, DM II, tabagismo e cardiopatias). São mostrados a média e a desviação padrão conforme apropriado. As variáveis qualitativas foram expressas em frequência (n) e porcentagem (\%).

Resultados: Um total de 70 pacientes foram avaliados na admissão; $54 \%$ eram mulheres, a idade média era de $69,1 \pm$ 12,24 anos. O tipo de acidente vascular cerebral predominante foi o isquêmico $(84,3 \%)$, sendo a maioria com peso adequado $(34,3 \%)$ seguido de sobrepeso $(31,4 \%)$. A maioria apresentou níveis desejáveis de colesterol total $(64,3 \%)$, níveis baixos de colesterol HDL (91,4\%), limite alto de colesterol $\operatorname{LDL}(47,1 \%)$ e níveis normais de triglicerídeos $(<150 \mathrm{mg} / \mathrm{dL}$, 38,5\%). Em relação aos demais fatores de 
de triglicéridos (< $150 \mathrm{mg} / \mathrm{dL}, 38,5 \%)$. Con respecto a los demás factores de riesgo, la mayoría tenía HTA $(81,4 \%)$.

Conclusión: en pacientes con antecedente de ACV, el factor de riesgo modificable predominante fue el de HTA, seguido de los niveles bajos de colesterol HDL. En este sentido, se resalta el papel del nutricionista para el cuidado de estos pacientes en el tratamiento de estos factores para su control o reducción, con el fin de evitar otro evento cerebrovascular.

Palabras clave: accidente cerebrovascular, obesidad, dislipidemia, alcoholismo, tabaquismo.

Centro Multidisciplinario de Investigaciones Tecnológicas, Universidad Nacional de Asunción. San Lorenzo, Paraguay.

2 Hospital Central de Policía "Rigoberto Caballero". Asunción, Paraguay. followed by low HDL cholesterol levels. Nutritionists play a key role in controlling and/or reducing these risk factors as part of the care of these patients, in order to avoid another cerebrovascular event.

Keywords: stroke, obesity, dyslipidemia, alcoholism, smoking. risco, a maioria apresentava hipertensão arterial (81,4\%).

Conclusão: Em pacientes com história de acidente vascular cerebral, o fator de risco modificável predominante foi o de pressão arterial elevada seguida de níveis baixos de colesterol HDL. Nesse sentido, destaca-se a atuação do nutricionista no cuidado a esses pacientes no tratamento desses fatores para seu controle e / ou redução a fim de evitar outro evento cerebrovascular.

Palavras-chave: acidente vascular cerebral, obesidade, dislipidemia, alcoolismo, tabagismo.

\section{INTRODUCCIÓN}

En general, las tasas de incidencia de accidentes cerebrovasculares (ACV), mortalidad y años de vida ajustados por discapacidad han disminuido durante las últimas 2 décadas en todo el mundo debido a las mejoras en los sistemas de atención médica y las opciones de tratamiento; sin embargo, con el crecimiento de la población, el envejecimiento, la mejora de la atención del ictus y el aumento de la prevalencia de factores de riesgo modificables, la cantidad de sobrevivientes de ictus y su carga global han aumentado ${ }^{(1)}$. Además, las tasas de incidencia de ictus a nivel mundial en los jóvenes y de mediana edad se han mantenido estables o incluso han aumentado en los últimos años, probablemente debido a la epidemia de obesidad. A medida que las enfermedades infecciosas han disminuido en todo el mundo, han aumentado las enfermedades no transmisibles $(\mathrm{ENT})^{(2)}$.

Las cuatro ENT principales (enfermedad cardiovascular [incluido el ACV], cáncer, diabetes y enfermedad pulmonar) comparten 4 factores de riesgo de estilo de vida: consumo de tabaco, dieta poco saludable, inactividad física y abuso de alcohol ${ }^{(3)}$. Por tanto, desde una perspectiva de salud pública, las acciones masivas sobre los factores de estilo de vida son los medios más rentables para la prevención de enfermedades. Tres de estos factores (consumo de tabaco, dieta y actividad física) están incorporados en las métricas de salud cardiovas-

cular de la American Heart Association (AHA) Life's Simple 7's, que abarcan 3 métricas médicas (presión arterial [PA], colesterol total, glucosa en sangre) y 4 métricas de comportamiento (tabaquismo, índice de masa corporal [IMC], actividad física y dieta $)^{(4)}$.

La mayoría de los accidentes cerebrovasculares se puede prevenir mediante la educación, el control de la PA y los cambios en el estilo de vida (dieta saludable, actividad física y control del hábito tabáquico) ${ }^{(5)}$. De hecho, esos 4 factores y la obesidad abdominal representaron el $82 \%$ y el $90 \%$ del riesgo atribuible a la población de $\mathrm{ACV}$ isquémico y hemorrágico en el estudio INTERSTROKE (factores de riesgo de ACV isquémico e intracerebral hemorrágico en 22 países) ${ }^{(5)}$. De manera similar, el estudio la de carga global de enfermedad mostró que el 90,5\% (intervalo de incertidumbre [IU] del $95 \%: 88,5-92,2$ ) de la carga global de ACV se atribuyó a factores de riesgo modificables ${ }^{(2)}$. Un estudio de modelado mostró que abordar múltiples factores de riesgo tiene beneficios aditivos para los efectos secundarios y la prevención. Específicamente, el uso de aspirina, estatinas y medicamentos antihipertensivos, combinado con la modificación de la dieta y el ejercicio, puede resultar en una reducción del $80 \%$ del riesgo acumulativo de eventos vasculares recurrentes ${ }^{(6)}$.

Aunque los beneficios de un estilo de vida saludable y el control de los factores de riesgo están bien documentados ${ }^{(7)}$, las intervenciones de control de los factores de riesgo no se han implementado de manera 
eficaz ${ }^{(8)}$ y los factores de riesgo siguen estando mal controlados entre los supervivientes de un $\mathrm{ACV}^{(9-11)}$. La prevención y el tratamiento del ACV requieren intervenciones complejas para abordar múltiples dominios (fisiológicos, psicológicos, sociales y ambientales) para enfocar el comportamiento humano y el control de los factores de riesgo vascular ${ }^{(12)}$.

Con base en lo mencionado anteriormente, es evidente la necesidad de realizar el seguimiento de este tipo de pacientes para monitorizar los factores de riesgo modificables con el fin de evitar otro evento cerebrovascular. Por tanto, el presente estudio tiene como objetivo evaluar la frecuencia de los factores de riesgo modificables en pacientes que han sufrido un ACV hospitalizados en el Hospital de Policía "Rigoberto Caballero" en el período de enero a junio de 2019.

\section{MATERIALES Y MÉTODOS}

Estudio observacional descriptivo realizado en 70 pacientes que han sufrido un ACV hospitalizados en el Hospital Central de la Policía "Rigoberto Caballero" en un período de 6 meses (enero a junio) de 2019. Se obtuvieron variables demográficas como edad y sexo; variables antropométricas como peso, talla e IMC; variables de factores de riesgo modificables como obesidad, colesterol total, colesterol LDL (lipoproteína de baja densidad), colesterol HDL (lipoproteína de alta densidad), HTA, diabetes mellitus tipo 2 (DM2), cardiopatías, arteriosclerosis, consumo de alcohol y consumo de tabaco.

Estos datos fueron tomados al momento del ingreso hospitalario de los pacientes que tenían historial de haber sufrido un ACV. El tipo de muestreo fue no probabilístico de casos consecutivos. Previa autorización de los directivos del hospital, se evaluaron a los participantes que desearon formar parte del estudio y firmaron un consentimiento informado. La evaluación de los pacientes se realizó a través del registro de las variables sociodemográficas, antropométricas, clínicas y de laboratorio de las fichas médicas; es decir, de fuente secundaria, ya que los pacientes estaban encamados. A través de una planilla elaborada en el programa Excel fueron cargados los datos. Después de comprobar la consistencia de la base de datos e identificar la distribución de las variables cuantitativas, se procedió al análisis de datos, expresando los promedios y desviaciones estándar (DE) según correspondiera. En el caso de las variables cualitativas, estas fueron expresadas en frecuencia (n) y porcentaje (\%). Todos los hallazgos fueron presentados en forma de tablas. Para el tratamiento de los datos se utilizó el programa SPSS 15.0 para Windows. Para llevar a cabo la investigación se tuvieron en cuenta los principios éticos de respeto a la persona, beneficencia y justicia.

El presente trabajo fue sometido y aprobado por Comité de Ética del Hospital, código número 03/2019.

\section{RESULTADOS}

De 113 pacientes, decidió participar un total de 70 pacientes que ingresaron al hospital con diagnóstico de $\mathrm{ACV}$, de los cuales $54 \%$ fue de sexo femenino con una edad en promedio de 69,17 $\pm 12,24$ años. La mayor parte de la población se encontraba en el grupo etario de 71 a 80 años $(35,7 \%)$. De acuerdo con el tipo de ACV, el 84,3\% ingresó por $\mathrm{ACV}$ de tipo isquémico (Tabla 1 ).

Tabla 1. Variables sociodemográficas y clínicas

\begin{tabular}{|l|c|}
\hline \multicolumn{1}{|c|}{ Variables } & Promedio \pm DE \\
\hline Edad (años) & $69,17 \pm 12,24$ \\
\hline Por grupo etario & $\mathbf{n}(\%)$ \\
\hline$-30-40$ & $2(3 \%)$ \\
\hline$-41-50$ & $3(4,3 \%)$ \\
\hline$-51-60$ & $8(11,4 \%)$ \\
\hline$-61-70$ & $23(32,3 \%)$ \\
\hline$-71-80$ & $25(35,7 \%)$ \\
\hline$-81-90$ & $8(11,4 \%)$ \\
\hline$-91-100$ & $1(1,4 \%)$ \\
\hline Sexo (\%) & \\
\hline- Femenino & $38(54 \%)$ \\
\hline- Masculino & $32(46 \%)$ \\
\hline Tipo de ACV & $\mathbf{n}(\%)$ \\
\hline- Isquémico & $59(84,3)$ \\
\hline- Hemorrágico & $11(15,7)$ \\
\hline
\end{tabular}

El promedio de IMC del total de la población fue de $26,8 \pm 3$,9. En la población femenina fue de $25,8 \pm 2,6$, mientras que en la población masculina fue de 27,2 \pm 3,7 . En cuanto al estado nutricional, se pudo constatar que el $34,3 \%$ se encontraba con un estado nutricional adecuado, seguido por un $31,4 \%$ con sobrepeso. En total, $48,5 \%$ de la población se encontraba con exceso de peso (Tabla 2). 
Tabla 2. Estado nutricional

\begin{tabular}{|l|c|}
\hline Estado nutricional & $\mathbf{n}(\%)$ \\
\hline - Bajo peso & $12(17,1 \%)$ \\
\hline - Adecuado & $24(34,3 \%)$ \\
\hline - Sobrepeso & $22(31,4)$ \\
\hline - Obesidad & $10(14,3 \%)$ \\
\hline - Obesidad I & $2(9 \%)$ \\
\hline IMC & Promedio \pm DE \\
\hline - General & $26,8 \pm 3,9$ \\
\hline IMC por sexo & \\
\hline - Femenino & $25,8 \pm 2,6$ \\
\hline - Masculino & $27,2 \pm 3,7$ \\
\hline
\end{tabular}

En cuanto a los niveles de colesterol, con respecto al colesterol total se ha constatado que el 35,7 \% tenía niveles por encima del valor deseable, 91,4 \% tenía niveles de colesterol HDL bajo y 55,7 \% tenía niveles de colesterol LDL por encima del valor deseable. En cuanto a los triglicéridos, $58,5 \%$ tenía niveles por encima del valor normal. La prevalencia de dislipidemia en la población fue de un 15,7 \% (Tabla 3).

Tabla 3. Niveles de colesterol y triglicéridos, y presencia de dislipidemia

\begin{tabular}{|l|l|l|}
\hline \multicolumn{1}{|c|}{ Variable } & \multicolumn{1}{|c|}{ Nivel/presencia } & \multicolumn{1}{c|}{ n (\%) } \\
\hline Colesterol total & Deseable & $45(64,3 \%)$ \\
& Límite elevado & $19(27,1 \%)$ \\
& Elevado & $6(8,6 \%)$ \\
\hline Colesterol HDL & Bajo & $64(91,4 \%)$ \\
& Deseable & $6(8,6 \%)$ \\
& Óptimo & $9(13 \%)$ \\
\hline Colesterol LDL & Deseable & $22(31,4 \%)$ \\
& Límite elevado & $33(47,1 \%)$ \\
& Elevado & $6(8,6 \%)$ \\
& Muy elevado & $0(0 \%)$ \\
\hline Triglicéridos & Normal & $27(38,5 \%)$ \\
& Límite alto & $20(28,5 \%)$ \\
& Alto & $21(30 \%)$ \\
& Muy alto & $2(3 \%)$ \\
\hline Dislipidemia & Sí & $11(15,7 \%)$ \\
& No & $59(84,3 \%)$ \\
\hline
\end{tabular}

En cuanto a los demás factores de riesgo, se ha observado que el 81,4 \% tenía HTA, 37,1 \% tenía DM2, 13
\% tenía cardiopatías, $16 \%$ consumía alcohol, 18,5 \% fumaba y $11,4 \%$ tenía aterosclerosis (Tabla 4 ).

Tabla 4. Factores de riesgo modificables asociados con ACV

\begin{tabular}{|l|c|c|}
\hline \multicolumn{2}{|c|}{ Factores de riesgo } & n (\%) \\
\hline HTA & Sí & $57(81,4 \%)$ \\
\hline DM2 & Sí & $26(37,1 \%)$ \\
\hline Cardiopatías & Sí & $9(13 \%)$ \\
\hline Alcohol & Sí & $11(16 \%)$ \\
\hline Tabaquismo & Sí & $13(18,5 \%)$ \\
\hline Aterosclerosis & Sí & $8(11,4 \%)$ \\
\hline
\end{tabular}

\section{DISCUSIÓN}

En este estudio se han evaluado los factores de riesgo modificables más comunes para el diagnóstico de ACV. A nivel sociodemográfico, se encontró que la edad promedio fue de 69 años. Este dato es relevante ya que se ha comprobado que la incidencia de ACV aumenta rápidamente con la edad, y se duplica cada década después de los 55 años $^{(13)}$. Por tanto, en cuanto a la prevención, es importante tener en cuenta que a partir de la edad mencionada se debe tener un plan de prevención para esta patología. Además, a medida que los pacientes que ya sufrieron un $\mathrm{ACV}$ van envejeciendo tienen más riesgo de sufrir otro evento.

Se ha encontrado que la mayor parte de la población fue de sexo femenino, aunque el riesgo es de $24 \%$ a $30 \%$ más alto en hombres, la cantidad anual de mujeres que sufren un $\mathrm{ACV}$ es mayor porque las mujeres tienen una mayor expectativa de vida ${ }^{(13)}$.

En el presente trabajo se evidenció que el 84,3\% de la población ingresó por ACV de tipo isquémico, resultado que concuerda con la literatura, en la que se registra que un $87 \%$ de los que sufren un $\mathrm{ACV}$ son de este tipo y que el $13 \%$ restante corresponde al tipo hemorrágico, cifra que se acerca al $15,7 \%$ encontrado de esa clasificación en este trabajo ${ }^{(14)}$.

La obesidad ha alcanzado proporciones epidémicas a nivel mundial, con al menos 2,8 millones de personas que mueren cada año como resultado de sobrepeso u obesidad. Una vez asociada con países de altos ingresos, la obesidad ahora también prevalece en países de bajos y medianos ingresos $^{(15)}$. En adultos, el riesgo de ACV aumenta casi linealmente a partir de un IMC de 20, y los adultos con un $\mathrm{IMC}>30 \mathrm{~kg} / \mathrm{m}^{2}$ tienen un riesgo $70 \%$ mayor de $\mathrm{ACV}$ en comparación con pacientes con un IMC $<25 \mathrm{~kg} / \mathrm{m}^{2(16)}$. 
Esta investigación constató un porcentaje considerable de pacientes con sobrepeso $(31,4 \%)$ y un $17,1 \%$ con obesidad, esta última cifra es baja en comparación con el $64 \%$ de pacientes obesos con ACV encontrado por Vicente y colaboradores ${ }^{(15)}$. No obstante, en conjunto estas cifras suman un $48,5 \%$ de pacientes con exceso de peso, cifra a tener en cuenta ya que su condición representa un riesgo para la enfermedad. En Paraguay, el $58 \%$ de los adultos tiene sobrepeso y obesidad $^{(17)}$. Llamativamente, no se encontró una frecuencia elevada de desnutrición, como es de esperar en este tipo de pacientes.

Según la $A H A^{(18)}$ y con respecto a los niveles de colesterol total, el $35,7 \%$ de los pacientes presentó niveles por encima de los valores deseables. En este sentido, un nivel alto de colesterol es un poderoso factor de riesgo de enfermedad coronaria, pero su papel en el ACV sigue siendo controvertido ${ }^{(19)}$. Aunque los estudios observacionales no han encontrado una asociación clara entre los niveles de colesterol y el ACV, se han informado asociaciones entre los niveles séricos elevados de colesterol total y un mayor riesgo de ACV isquémico ${ }^{(20)}$. Un estudio reciente mostró que el colesterol sérico por debajo de $160 \mathrm{mg} / \mathrm{dL}$ ya constituía un factor de riesgo para un ACV hemorrágico ${ }^{(21)}$.

El 91,4\% de los pacientes presentó niveles de colesterol HDL bajo. Este porcentaje supera al encontrado por Olamoyegun y colaboradores, quienes encontraron un 74,5\% de pacientes con ACV y HDL bajo ${ }^{(22)}$.

La dislipidemia es significativamente mayor en el $\mathrm{ACV}$ isquémico en comparación con el hemorrágico. En este trabajo se evidenció un $15,7 \%$ de prevalencia de dislipidemia. La reducción del colesterol HDL es la fracción más frecuente de las anormalidades lipídicas ${ }^{(22)}$.

En la presente investigación se ha encontrado que la mayor parte de la población tenía niveles de colesterol LDL en un límite elevado ( $145 \mathrm{mg} / \mathrm{dL})$, esta cifra supera al promedio que Wang y colaboradores encontraron en su estudio, en el que el promedio de todos los pacientes con ACV de LDL fue de 93,61 mg/dL. Estudios recientes han demostrado que la reducción de colesterol LDL podría provocar una reducción del riesgo del $16 \%, 21 \%$ y $23 \%$ en todos los $\mathrm{ACV}, \mathrm{ACV}$ isquémico y primer $\mathrm{ACV}$ isquémico no mortal, respectivamente ${ }^{(23)}$. Por tanto, la directriz de ACV de 2014 de la American Heart Association/American Stroke Association (AHA/ASA) recomendó que se usen estatinas de alta intensidad para reducir el riesgo de ACV y eventos cardiovasculares entre pacientes con ACV isquémico o ataque isquémico transitorio ${ }^{(24)}$.
En total, el 61,5\% de los pacientes presentó niveles de triglicéridos por encima del valor considerado normal. Esta cifra supera ampliamente a lo encontrado por Lee y colaboradores, quienes encontraron un total de $30 \%$ de pacientes con niveles altos de triglicéridos. Se ha demostrado que los niveles elevados de triglicéridos además de niveles de colesterol HDL bajos incrementan el riesgo de la ocurrencia de un $\mathrm{ACV}^{(25)}$.

Con respecto a la variable de $\mathrm{PA}$, el $81,4 \%$ de los pacientes presentó HTA. En el estudio INTERSTROKE, la HTA fue el factor de riesgo de ACV más importante: se usó una definición de HTA que incluía tanto un historial de HTA como una medición de la PA de 160/90 mm $\mathrm{Hg}$; y el riesgo atribuible a la población o la proporción de ACV en la población atribuible a HTA fue del $54 \%$. Aunque este fue un estudio de casos y controles $y$, por tanto, las mediciones de la PA probablemente se vieron confundidas por un ACV reciente, los resultados aún implican un efecto importante de la PA sobre el riesgo de $\mathrm{ACV}$ y son consistentes con otros estudios. El efecto de la PA también fue mayor para el ACV hemorrágico que para el isquémico ${ }^{(5)}$.

Del total de la población estudiada, 37,1 \% tenía DM2. La glucemia no controlada pone a los sujetos en riesgo de sufrir $\mathrm{ACV}$ isquémicos y hemorrágicos. Existen patrones clínicos específicos de ACV isquémico en personas con diabetes. Por ejemplo, las personas con diabetes tienen más probabilidades de tener debilidad en las extremidades y disartria como signos de infarto cerebral lacunar, en comparación con aquellos sin diabetes. Ningún ensayo clínico importante ha examinado estrategias específicas de prevención del ACV en personas con diabetes ${ }^{(26)}$. La evidencia es escasa en la prevención secundaria del ACV. La mayoría de los datos disponibles se basa en estudios centrados en la prevención primaria del $\mathrm{ACV}^{(27)}$. El control agresivo de la glucosa a través del cambio de estilo de vida o medicamentos y la modificación de otros factores de riesgo asociados (como la presión sanguínea y la dislipidemia) son pasos críticos hacia la prevención efectiva del $\mathrm{ACV}^{(28)}$.

El $13 \%$ de los pacientes ingresó al hospital con cardiopatías. La contribución de las complicaciones cardíacas a la mortalidad de los pacientes con ACV es variable entre los estudios, que van del $12,5 \%$ al $22,7 \%{ }^{(29)}$.

En cuanto al consumo de alcohol y tabaco, del total de pacientes, $16 \%$ y $18,5 \%$ lo consumían, respectivamente. Por una parte, el consumo excesivo de alcohol aumenta el riesgo relativo de cualquier $\mathrm{ACV}^{(30)}$. Por otra parte, se estima que el consumo de tabaco es 
responsable del $12 \%$ al $37 \%$ de todos los eventos de $\mathrm{ACV}$ y, por tanto, es una de las principales causas prevenibles ${ }^{(31)}$. Dejar de fumar puede ser particularmente benéfico para los pacientes que ya han tenido un $\mathrm{ACV}$ isquémico porque tienen un riesgo muy alto de futuros eventos cardiovasculares ${ }^{(32)}$.

El 11,4 \% de los pacientes presentó aterosclerosis evaluada por ecografía Doppler. En este sentido, los pacientes que sobreviven a un ACV tienen un mayor riesgo de eventos vasculares recurrentes ${ }^{(33)}$. Los mecanismos subyacentes a los eventos apenas se comprenden. Un estudio reciente sugiere que la aterosclerosis aumentada por ACV se induce a través de alarmas liberadas por el cerebro, que conducen a inflamación vascular sistémica y formación de placa ${ }^{(34)}$.

Como hemos visto, los factores de riesgo modificables para que ocurra un $\mathrm{ACV}$ son los mismos que persisten luego de la ocurrencia del mismo y pueden desencadenar otro. Todos los factores de riesgo que hemos evaluado (a excepción del sexo y la edad) son modificables, y la intervención nutricional es de suma importancia ya sea para revertir las cifras fuera de rango o hábitos no saludables, o para prevenir que esto ocurra.

Un paciente que ha sufrido un ACV requiere de especial atención por un equipo multidisciplinario que le permita desenvolverse lo más autónomamente posible dentro de sus posibilidades, de modo que se evite que sufra un nuevo evento.

Como limitación del presente estudio se puede mencionar que, al ser descriptivo, no se realizaron relaciones entre las variables de estudio. Una de las fortalezas de este estudio es que es el primero que evalúa, en nuestra institución, la prevalencia de factores de riesgo en pacientes que ya han sufrido un ACV. Dentro de estos factores, los modificables son los que se pueden controlar y, por tanto, la identificación de estos es primordial tanto en la prevención como en el tratamiento de un ACV para prevenir un nuevo evento.

\section{CONCLUSIÓN}

En los pacientes que han sufrido un ACV persisten los factores de riesgo modificables, especialmente la HTA y el colesterol HDL bajo. Además, se evaluó la proporción de exceso de peso, lo cual constituye un factor de riesgo más para esta patología. En este sentido, el papel del nutricionista es fundamental para revertir estos parámetros ya que están estrechamente relacionados con la alimentación y es por ello que deben estar presentes dentro del equipo multidisciplinario en el tratamiento de este tipo de pacientes.

\section{PUNTOS CLAVE}

- El ACV es una patología de alta mortalidad y que va creciendo en términos de prevalencia e incidencia.

- La mayoría de los pacientes que han sufrido un ictus aún presenta los factores de riesgo modificables, razón por la que estas deben ser tratadas por un nutricionista.

\section{Declaración de relevancia clínica}

El ACV es la segunda causa de muerte y la principal causa de discapacidad en todo el mundo. La carga del $\mathrm{ACV}$ varía considerablemente entre los países, pero los países de bajos ingresos son los más afectados.

Los principales factores de riesgo modificables son la hipertensión arterial (HTA), la diabetes mellitus, el consumo de tabaco y la hiperlipidemia, así como los factores relacionados con el estilo de vida, como la obesidad, la mala alimentación/nutrición y la inactividad física.

Un paciente que ya ha sufrido un ictus también debe ser tratado en cuanto a estos factores que persisten en él y cuyo tratamiento podría prevenir otro evento cerebrovascular.

Habiendo mencionado esto, el presente trabajo basa su relevancia clínica en conocer el estado de pacientes que sufrieron un ictus y en los factores de riesgo mencionados; y también resalta el rol del nutricionista en cuanto al tratamiento de estos pacientes, ya que estos factores están relacionados con la alimentación.

\section{Declaración de autoría}

Todas las autoras contribuyeron a la concepción y ejecución del presente trabajo de investigación, así como en la recolección de datos, su procesamiento y escritura del manuscrito previa revisión de todas las partes.

\section{Conflicto de intereses}

Las autoras declaran no tener conflictos de intereses.

\section{Fuente de financiación}

El estudio no tuvo financiación.

\section{Referencias bibliográficas}

1. Feigin VL, Norrving B, Mensah GA. Global Burden of Stroke. Circ Res. 2017;120(3):439-448. doi: 10.1161/ CIRCRESAHA.116.308413

2. Feigin VL, Roth GA, Naghavi M, Parmar P, Krishnamurthi $\mathrm{R}$, Chugh $\mathrm{S}$, et al. Global burden of stroke and risk factors in 
188 countries, during 1990-2013: a systematic analysis for the Global Burden of Disease Study 2013. Lancet Neurol. 2016;15(9):913-924. doi: 10.1016/S1474-4422(16)30073-4

3. Beaglehole R, Bonita R, Horton R, Adams C, Alleyne G, Asaria $\mathrm{P}$, et al. Priority actions for the non-communicable disease crisis. Lancet. 2011;377(9775):1438-47. doi: 10.1016/S0140-6736(11)60393-0

4. Lloyd-Jones DM, Hong Y, Labarthe D, Mozaffarian D, Appel LJ, Van Horn L, et al. Defining and setting national goals for cardiovascular health promotion and disease reduction: the American Heart Association's strategic Impact Goal through 2020 and beyond. Circulation. 2010;121(4):586-613. doi: 10.1161/CIRCULATIONAHA.109.192703

5. O’Donnell M, Xavier D, Diener C, Sacco R, Lisheng L, Zhang $\mathrm{H}$, etal.Rationale and design of INTERSTROKE: aglobal casecontrol study of risk factors for stroke. Neuroepidemiology. 2010;35(1):36-44. doi: 10.1159/000306058

6. Hackam DG, Spence JD. Combining multiple approaches for the secondary prevention of vascular events after stroke: a quantitative modeling study. Stroke. 2007;38(6):1881-5. doi: 10.1161/STROKEAHA.106.475525

7. Coutts SB, Wein TH, Lindsay MP, Buck B, Cote R, Ellis P, et al. Canadian Stroke Best Practice Recommendations: secondary prevention of stroke guidelines, update 2014. Int J Stroke. 2015;10(3):282-91. doi: 10.1111/ijs.12439

8. Lichtman JH, Naert L, Allen NB, Watanabe E, Jones SB, Barry LC, et al. Use of antithrombotic medications among elderly ischemic stroke patients. Circ Cardiovasc Qual Outcomes. 2011;4(1):30-8. doi: 10.1161/ CIRCOUTCOMES.109.850883

9. Razmara A, Ovbiagele B, Markovic D, Towfighi A. Patterns and Predictors of Blood Pressure Treatment, Control, and Outcomes among Stroke Survivors in the United States. J Stroke Cerebrovasc Dis. 2016;25(4):857-65. doi: 10.1016/j. jstrokecerebrovasdis.2015.12.027

10. Lin MP, Ovbiagele B, Markovic D, Towfighi A. "Life's Simple 7" and Long-Term Mortality After Stroke. J Am Heart Assoc. 2015;4(11):e001470. doi: 10.1161/JAHA.114.001470

11. Heuschmann PU, Kircher J, Nowe T, Dittrich R, Reiner Z, Cifkova $\mathrm{R}$, et al. Control of main risk factors after ischaemic stroke across Europe: data from the stroke-specific module of the EUROASPIRE III survey. Eur J Prev Cardiol. 2015;22(10):1354-62. doi: 10.1177/2047487314546825

12. Redfern J, McKevitt C, Wolfe CD. Development of complex interventions in stroke care: a systematic review. Stroke. 2006;37(9):2410-9. doi: 10.1161/01. STR.0000237097.00342.a9

13. Ovbiagele B, Nguyen-Huynh MN. Stroke epidemiology: advancing our understanding of disease mechanism and therapy. Neurotherapeutics. 2011;8(3):319-29. doi: 10.1007/ s13311-011-0053-1

14. Chauhan G, Debette S. Genetic Risk Factors for Ischemic and Hemorrhagic Stroke. Curr Cardiol Rep. 2016;18(12):124. doi: $10.1007 / \mathrm{s} 11886-016-0804-\mathrm{z}$
15. Obesity [internet]. Génova: World Health Organization; 2017 [citado el 10 de agosto de 2021]. Disponible en: http:// www.who.int/features/factfiles/obesity/en/

16. Vicente VS, Cabral NL, Nagel V, Guesser VV, Safanelli J. Prevalence of obesity among stroke patients in five Brazilian cities: a cross-sectional study. Arq Neuropsiquiatr. 2018;76(6):367-372. doi: 10.1590/0004-282X20180053

17. Unas 2.600 muertes al año se asocian a la obesidad en Paraguay. [Internet]. Ministerio de Salud Pública y Bienestar Social; 2011 [consultado el 10 de agosto de 2021]. Disponible en: https://www.mspbs.gov.py/portal/18105/unas-2600-muertes-al-antildeo-se-asocian-a-la-obesidad-en-paraguay.html

18. Lloyd-Jones DM, Hong Y, Labarthe D, Mozaffarian D, Appel LJ, Van Horn L, et al. Defining and setting national goals for cardiovascular health promotion and disease reduction: the American Heart Association's strategic Impact Goal through 2020 and beyond. Circulation. 2010;121(4):586-613. doi: 10.1161/CIRCULATIONAHA.109.192703

19. Nam BH, Kannel WB, D’Agostino RB. Search for an optimal atherogenic lipid risk profile: from the Framingham Study. Am J Cardiol. 2006;97(3):372-5. doi: 10.1016/j.amjcard.2005.08.055

20. Suzuki K, Izumi M, Sakamoto T, Hayashi M. Blood pressure and total cholesterol level are critical risks especially for hemorrhagic stroke in Akita, Japan. Cerebrovasc Dis. 2011;31(1):100-6. doi: 10.1159/000321506

21. Zhao W, An Z, Hong Y, Zhou G, Guo J, Zhang Y, et al. Low total cholesterol level is the independent predictor of poor outcomes in patients with acute ischemic stroke: a hospitalbased prospective study. BMC Neurol. 2016;16:36. doi: $10.1186 / \mathrm{s} 12883-016-0561-\mathrm{z}$

22. Olamoyegun MA, Akinlade AT, Fawale MB, Ogbera AO. Dyslipidaemia as a risk factor in the occurrence of stroke in Nigeria: prevalence and patterns. Pan Afr Med J. 2016;25:72. doi: 10.11604/pamj.2016.25.72.6496

23. Ji R, Liu G, Shen H, Wang Y, Li H, Peterson E, et al. Persistence of secondary prevention medications after acute ischemic stroke or transient ischemic attack in Chinese population: data from China National Stroke Registry. Neurol Res. 2013;35(1):29-36. doi: 10.1179/1743132812Y.0000000107

24. Wang CJ, Wang YL, Li ZX, Wang YJ. The Management of LDL Cholesterol and Predictors of Goal Achievement in Stroke Patients in China: A Cross-Sectional Study. CNS Neurosci Ther. 2016;22(7):577-83. doi: 10.1111/cns.12543

25. Lee JS, Chang PY, Zhang Y, Kizer JR, Best LG, Howard BV. Triglyceride and HDL-C Dyslipidemia and Risks of Coronary Heart Disease and Ischemic Stroke by Glycemic Dysregulation Status: The Strong Heart Study. Diabetes Care. 2017;40(4):529-537. doi: 10.2337/dc16-1958

26. Karapanayiotides T, Piechowski-Jozwiak B, van Melle G, Bogousslavsky J, Devuyst G. Stroke patterns, etiology, and prognosis in patients with diabetes mellitus. Neurology. 2004;62(9):1558-62. doi: 10.1212/01. wnl.0000123252.55688.05 
27. Boehme AK, Esenwa C, Elkind MS. Stroke Risk Factors, Genetics, and Prevention. Circ Res. 2017;120(3):472-495. doi: 10.1161/CIRCRESAHA.116.308398

28. Chen R, Ovbiagele B, Feng W. Diabetes and Stroke: Epidemiology, Pathophysiology, Pharmaceuticals and Outcomes. Am J Med Sci. 2016;351(4):380-6. doi: 10.1016/j. amjms.2016.01.011

29. Kenmogne-Domning GH, Kamtchum-Tatuene J, Noumegni SR, Fokoua-Dongmo CM, Zafack JG, Noubiap JJ. Cardiac complications after stroke: protocol for a systematic review and meta-analysis. BMJ Open. 2018;8(5):e021416. doi: 10.1136/bmjopen-2017-021416

30. Patra J, Taylor B, Irving H, Roerecke M, Baliunas D, Mohapatra $S$, et al. Alcohol consumption and the risk of morbidity and mortality for different stroke types--a systematic review and meta-analysis. BMC Public Health. 2010;10:258. doi: $10.1186 / 1471-2458-10-258$
31. Bonita R, Scragg R, Stewart A, Jackson R, Beaglehole R. Cigarette smoking and risk of premature stroke in men and women. Br Med J (Clin Res Ed). 1986;293(6538):6-8. doi: 10.1136/bmj.293.6538.6

32. Epstein KA, Viscoli CM, Spence JD, Young LH, Inzucchi SE, Gorman M, et al. Smoking cessation and outcome after ischemic stroke or TIA. Neurology. 2017;89(16):1723-1729. doi: 10.1212/WNL.0000000000004524

33. Putaala J, Haapaniemi E, Metso AJ, Metso TM, Artto V, Kaste $\mathrm{M}$, et al. Recurrent ischemic events in young adults after firstever ischemic stroke. Ann Neurol. 2010;68(5):661-71. doi: 10.1002/ana.22091

34. Rust R, Hofer AS, Schwab ME. Stroke Promotes Systemic Endothelial Inflammation and Atherosclerosis. Trends Mol Med. 2018;24(7):593-595. doi: 10.1016/j.molmed.2018.04.008 